Orthopäde 2022 $\cdot 51: 403-409$

https://doi.org/10.1007/s00132-021-04197-3

Angenommen: 17. November 2021

Online publiziert: 3. Januar 2022

() Der/die Autor(en) 2022

\section{Einfluss der COVID-19-Pandemie auf die physiotherapeutische und ärztliche Nachsorge nach Rekonstruktionen des vorderen Kreuzbandes}

\author{
Benjamin Bartek · Tobias Jung · Antonia Schwiedernoch - Carsten Perka · \\ Yannick Palmowski \\ Center for Musculoskeletal Surgery, Charité - Universitätsmedizin Berlin, Humboldt-Universität zu Berlin, \\ Berlin Institute of Health, Berlin, Deutschland
}

Hintergrund: Ziel der Studie war es, den Einfluss der COVID-19-Pandemie auf die physiotherapeutische und ärztliche Nachsorge nach Rekonstruktion des vorderen Kreuzbandes (VKB) zu untersuchen.

Methoden: Insgesamt wurden 116 Patienten (72 Männer und 44 Frauen) eingeschlossen, die zwischen September 2019 und Dezember 2020 eine Rekonstruktion des VKB erhalten hatten. Die Patienten wurden in eine 2019 vor der COVID-

19( „„Coronavirus disease" 2019)-Pandemie operierte Patientengruppe sowie eine 2020 operierte, von der COVID-19-Pandemie unmittelbar betroffene Patientengruppe eingeteilt. Anhand eines standardisierten Fragebogens wurden die Auswirkungen der Pandemie auf die ärztliche und physiotherapeutische Nachsorge sowie auf die Dauer der postoperativen Arbeitsunfähigkeit erhoben. Ergänzend erfolgte eine Auswertung des Bewegungsumfanges des operierten Knies 3 Monate postoperativ.

Ergebnisse: Es zeigte sich bei den 2020 operierten Patienten eine deutliche Tendenz zu einem höheren Anteil an Streckdefiziten $\geq 5^{\circ}(18,8 \%$ vs. $4,3 \%, p=0,097)$ oder einer maximalen Beugefähigkeit von $<120^{\circ}(23,3 \%$ vs. $10 \%, p=0,197) 3$ Monate postoperativ, die allerdings nicht signifikant war. In der physiotherapeutischen und ärztlichen Nachsorge berichteten die 2020 operierten Patienten von signifikant längeren Wartezeiten auf Termine, späteren Zeitpunkten des ersten postoperativen Termins, einer höheren Anzahl vergeblicher Terminanfragen sowie einer höheren Anzahl abgesagter Termine. 34,9\% der 2020 operierten Patienten gaben an, die Dauer ihrer postoperativen Arbeitsunfähigkeit durch die Möglichkeiten zur Arbeit im Homeoffice verkürzt haben zu können. Pandemiebedingte alternative Behandlungsangebote wurden den Patienten von 13,3\% der Physiotherapeuten sowie $12,2 \%$ der Ärzte angeboten.

Schlussfolgerung: Obwohl die physiotherapeutische und ärztliche Behandlung von keinem „Lockdown" betroffen war, kam es aufgrund der Pandemie zu signifikanten Einschränkungen, welche sich auch in einer deutlichen Tendenz zu schlechteren klinischen Ergebnissen niederschlagen. Es besteht daher Bedarf an einem weiteren Ausbau alternativer Therapieoptionen, die bislang nur von 12-13\% der Praxen zur Verfügung gestellt werden und mutmaßlich auch für andere Erkrankungen von ähnlicher Bedeutung sind.

\section{Schlüsselwörter}

VKB-Rekonstruktion · "Coronavirus disease“ 2019 · Kniegelenk · Physiotherapeuten · SARS-CoV-2 


\section{Einleitung}

Mit einer jährlichen Inzidenz von über 40.000 Rupturen des vorderen Kreuzbandes (VKB) deutschlandweit sowie etwa 1/1000 in der Altersgruppe 15-25 Jahre gehört die VKB-Ruptur zu den häufigsten Sportverletzungen junger Patienten [1-3]. Insbesondere bei jungen Patienten steht laut aktueller S1-Leitlinie aufgrund des hohen funktionellen Anspruchs sowie des langfristig erhöhten Arthroserisikos bei VKB-Insuffizienz die operative Therapie im Vordergrund, die in der Regel durch eine Rekonstruktion mit autologer Sehnenplastik erfolgt $[4,5]$.

Für ein optimales postoperatives Outcome mit langfristig möglichst vollständiger Wiederherstellung der Funktionalität und Belastbarkeit ist die physiotherapeutische Nachsorge von entscheidender Bedeutung. Diese erfolgt üblicherweise entsprechend standardisierter Nachbehandlungsschemata. Die Relevanz einer konsequenten Physiotherapie mit frühzeitigem Beginn der Therapie, kontinuierlicher Wahrnehmung aller Termine und regelmäßiger Kontrolle des Fortschritts ist in Studien belegt $[6,7]$.

Seit Ende 2019 verbreitet sich die "coronavirus disease" 2019 (COVID-19) weltweit. In Deutschland wurde zum Infektionsschutz ab dem 11.03.2021 an die Bevölkerung appelliert, Kontakte zu beschränken. Bereits kurz darauf trat $a b$ dem 22.03.2020 der erste "Lockdown" in Kraft und führte zu deutlichen Einschränkungen in vielen Bereichen des öffentlichen Lebens [8]. Bisher ist unseres Wissens ungeklärt, welche Auswirkungen die COVID-19-Pandemie sowie die damit verbundenen Kontaktbeschränkungen auf die postoperative Nachsorge hatten und auch die Folgen für das klinische Outcome sind bislang nicht bekannt. Um dies zu untersuchen, führten wir in der vorliegenden Studie daher eine Befragung von Patienten durch, welche während oder direkt vor der Pandemie eine Rekonstruktion des VKB erhielten und ergänzten dies durch

\section{Abkürzungen}

ANOVA Varianzanalyse

COVID-19 „Coronavirus disease“ 2019

VKB Vorderes Kreuzband postoperativ erfasste klinische Untersuchungsbefunde. Ziel war es, zu ermitteln, ob die im Rahmen der Pandemie getroffenen Maßnahmen einen Einfluss auf die physiotherapeutische und ärztliche Nachsorge nach VKB-Rekonstruktionen hatten, sowie ob es hierdurch zu einer Beeinträchtigung der klinischen Ergebnisse kam. Dabei sollte die VKB-Rekonstruktion in erster Linie als Beispiel für eine Vielzahl an orthopädischen und auch sonstigen Erkrankungen dienen, bei denen eine enge ärztliche und physiotherapeutische Betreuung erforderlich ist.

\section{Material und Methoden}

\section{Ethikvotum}

Die Durchführung der Studie wurde von der Ethikkommission der Charité Universitätsmedizin Berlin genehmigt (EA2/072/21).

\section{Patienten}

Für die Durchführung dieser retrospektiven Kohortenstudie wurden alle volljährigen Patienten kontaktiert, die im Zeitraum zwischen September 2019 und April 2020 am Centrum für Muskuloskeletale Chirurgie der Charité - Universitätsmedizin Berlin eine Rekonstruktion des VKB erhalten haben. Diese Patienten wurden je nach Operationszeitpunkt in zwei Gruppen eingeteilt: eine 2019 operierte Gruppe, welche mindesten 12 Wochen Physiotherapie vor Inkrafttreten des ersten "Lockdowns" erhielt, sowie eine 2020 operierte Gruppe, welche während der ersten 3 postoperativen Monate von Einschränkungen durch die COVID-19-Pandemie sowie den dadurch bedingten "Lockdown" betroffen war.

\section{Datenerhebung}

Bei Einverständnis zur Studienteilnahme erfolgte eine telefonische Befragung der Teilnehmer sowie eine Auswertung der im Rahmen der postoperativen Nachkontrollen routinemäßig erfassten klinischen Untersuchungsbefunde. Im Rahmen der telefonischen Datenerhebung wurden die Teilnehmer anhand eines vorher definierten standardisierten Fragebogens zu ihren
Erfahrungen bei der postoperativen physiotherapeutischen und ärztlichen Nachsorge in Zeiten der COVID-19-Pandemie befragt. Erfragt wurden dabei (jeweils separat für die ärztliche und physiotherapeutische Nachsorge) die Wartezeit auf einen Termin, der Zeitraum zwischen Operation und Wahrnehmen des ersten Termins, die Anzahl vergeblicher Terminanfragen, die Anzahl selbst oder durch die jeweilige Praxis abgesagter Termine, Einschränkungen der Behandlung durch die Pandemie, aufgrund der Pandemie eingeführte alternative Therapieoptionen, generelle Zufriedenheit mit der Behandlung (auf einer Skala von 1-10) sowie die Einschätzung des Einflusses der Pandemie auf die Qualität der Nachsorge (auf einer Skala von 1-10). Zudem wurde erfragt, wie lange die Patienten postoperativ krankgeschrieben waren, ob die Dauer der Arbeitsunfähigkeit aufgrund von Homeoffice im Rahmen der Pandemie verkürzt werden konnte und ob sich die Patienten erneut in einer vergleichbaren Situation operieren lassen würden. Ergänzend zu den telefonisch erhobenen Daten wurde bei allen Patienten der im Rahmen der routinemäßigen Nachkontrollen nach klinischer Untersuchung dokumentierte Bewegungsumfang (Neutral-Null-Methode) des operierten Knies 3 Monate postoperativ erfasst und es wurde der Anteil an Patienten mit einem Streck- oder Beugedefizit errechnet. Hierbei wurden ein Streckdefizit von $\geq 5^{\circ}$ oder eine maximale Beugefähigkeit von $<120^{\circ}$ als relevante Bewegungseinschränkung gewertet. Die Befragung und die Auswertung der klinischen Untersuchungsbefunde erfolgte im April 2021, sodass sich zu diesem Zeitpunkt alle Patienten bereits seit mindestens 12 Wochen in der postoperativen Nachbehandlung befanden.

\section{Statistische Auswertung}

Die statistische Auswertung erfolgte mit dem Programm SPSS in der Version 27 (IBM, New York, NY, USA). Zur Untersuchung der Unterschiede zwischen beiden Gruppen auf Signifikanz wurden bei parametrischen Variablen der Student's T-Test, bei nichtparametrischen Variablen der MannWhitney-U-Test sowie bei binären Variablen der Chi-Quadrat Test genutzt. Für den 
Tab. 1 Vergleich der Umfrageergebnisse zur physiotherapeutischen Nachbehandlung der zwischen 2019 und 2020 operierten Patienten

\begin{tabular}{|l|l|l|l|l|l|}
\hline & $\mathbf{2 0 1 9}$ & $\mathbf{2 0 2 0}$ & \multicolumn{1}{l|}{$p$} \\
\hline Wartedauer auf Termin (Wochen) & Mittelwert & SD & Mittelwert & SD \\
\hline Zeitpunkt des ersten Termins (Wochen postoperativ) & 1,09 & 0,29 & 2,57 & 5,75 & 0,022 \\
\hline Anzahl vergeblicher Terminanfragen & 1,15 & 0,44 & 1,88 & 1,82 & 0,001 \\
\hline Anzahl durch Physiotherapie abgesagter Termine & 0,26 & 1,14 & 0,94 & 1,52 & 0,010 \\
\hline Anzahl selbst abgesagter Physiotherapietermine & 0,32 & 0,91 & 1,16 & 1,67 & 0,001 \\
\hline Zufriedenheit mit der Behandlung (1= sehr unzufrieden, $10=$ sehr zufrieden) & 0,12 & 0,41 & 0,90 & 1,72 & 0,000 \\
\hline SD Standardabweichung & 8,56 & 1,16 & 7,83 & 1,66 & 0,021 \\
\hline
\end{tabular}

Tab. 2 Vergleich der Umfrageergebnisse zur ärztlichen Nachbehandlung der zwischen 2019 und 2020 operierten Patienten

\begin{tabular}{|l|l|l|l|l|l|}
\hline & \multicolumn{2}{l|}{$\mathbf{2 0 2 0}$} & \multicolumn{1}{l|}{$p$} \\
\hline Wartedauer auf Termin (Wochen) & $\mathbf{2 0 1 9}$ & Mittelwert & SD & Mittelwert & SD \\
\hline Zeitpunkt des ersten Termins (Wochen postoperativ) & 1,30 & 1,02 & 2,28 & 1,81 & 0,000 \\
\hline Anzahl vergeblicher Terminanfragen & 1,42 & 1,28 & 2,38 & 1,81 & 0,002 \\
\hline Anzahl durch Orthopäden abgesagter Termine & 0,03 & 0,17 & 0,32 & 0,65 & 0,000 \\
\hline Anzahl selbst abgesagter Orthopädietermine & 0,03 & 0,17 & 0,38 & 0,90 & 0,001 \\
\hline Zufriedenheit mit der Behandlung (1 = sehr unzufrieden, $10=$ sehr zufrieden) & 0,00 & 0,00 & 0,29 & 0,60 & 0,000 \\
\hline SD Standardabweichung & 7,48 & 2,03 & 7,16 & 1,81 & 0,400 \\
\hline
\end{tabular}

Vergleich von > 2 Gruppen wurde eine einfaktorielle Varianzanalyse (ANOVA) durchgeführt.

\section{Ergebnisse}

\section{Studienkollektiv}

Insgesamt erhielten im Zeitraum zwischen September 2019 und Dezember 2020168 Patienten eine Rekonstruktion des VKB, von denen 8 aufgrund von Minderjährigkeit ausgeschlossen wurden. Von den verbleibenden Patienten konnten 116 (72 Männer und 44 Frauen) kontaktiert werden und erklärten sich zur Studienteilnahme bereit. Von diesen wurden 33 (23 Männer und 10 Frauen) 2019 vor der COVID-19-Pandemie operiert, wohingegen 83 (49 Männer und 34 Frauen) Patienten 2020 operiert wurden und somit zumindest Teile der Nachsorge während der COVID-19-Pandemie absolvierten. Das Durchschnittsalter lag bei 33,9 Jahren (2019: 34 Jahre, 2020: 33,8 Jahre). Entnahmestelle für das Sehnentransplantat war in der Regel der M. semitendinosus. Daneben wurden in zwei Fällen (davon $1 \mathrm{im} \mathrm{Jahr}$ 2019 und $1 \mathrm{im}$ Jahr 2020) die Sehnen des $M$. semitendinosus und $M$. gracillis sowie in 13 Fällen (davon 4 im Jahr 2019 und 9 im Jahr 2020) ein Transplantat aus der
Quadrizepssehne genutzt. Alle Operationen während des untersuchten Zeitraums wurden von den gleichen drei erfahrenen Operateuren in einem stationären Setting durchgeführt. Die Nachbehandlung erfolgte anhand standardisierter Nachbehandlungsschemata, die während des untersuchten Zeitraums nicht verändert wurden.

Auswirkungen auf die physiotherapeutische Nachsorge

Bei der Befragung zur physiotherapeutischen Nachbehandlung zeigten sich hinsichtlich aller erfragten Items signifikante Unterschiede zwischen den 2019 und 2020 operierten Patienten (- Tab. 1).

\section{Auswirkungen auf die ärztliche Nachsorge}

Bei der Befragung zur ärztlichen Nachbehandlung zeigten sich hinsichtlich der Fragen zu Terminvereinbarung und -wahrnehmung signifikante Unterschiede zwischen den 2019 und 2020 operierten Patienten (• Tab. 2).
Einfluss auf das postoperative Bewegungsausmaß

Hinsichtlich des Bewegungsumfanges 3 Monate postoperativ zeigte sich bei den 2020 operierten Patienten eine nicht signifikante Tendenz zu einem häufigeren Streckdefizit $\geq 5^{\circ}$ als vor der COVID-19Pandemie $(4,3 \%$ vs. $18,8 \%, p=0,097)$. Zudem bestand eine Tendenz zu einem häufigeren Beugedefizit mit einer maximalen passiven Beugefähigkeit von $<120^{\circ}$, welche ebenfalls nicht signifikant war ( $10 \%$ vs. $23,3 \%, p=0,197)$.

\section{Einfluss der COVID-19-Pandemie auf die Dauer der Arbeitsunfähigkeit}

Einer von 33 Patienten 2019 (2,9\%) sowie 29/82 Patienten 2020 (34,9\%) gaben an, dass sich durch die Pandemie die Dauer ihrer postoperativen Arbeitsunfähigkeit verkürzt habe. Im Durchschnitt betrug die Dauer der Arbeitsunfähigkeit 2019 7,77 $( \pm 6,10)$ Wochen und $20208,53( \pm 10,37)$ Wochen $(p=0,688)$. Bei genauerer Unterteilung des Jahres 2020 in einzelne Tertiale zeigt sich ein signifikanter Unterschied in der Dauer der Arbeitsunfähigkeit mit 13,04 $( \pm 3,68)$ Wochen im 1. Tertial, $7,10( \pm 4,00)$ Wochen im 2. Tertial sowie $6,54( \pm 4,63)$ Wochen im 3. Tertial $(p=0,048)$. Eine Über- 


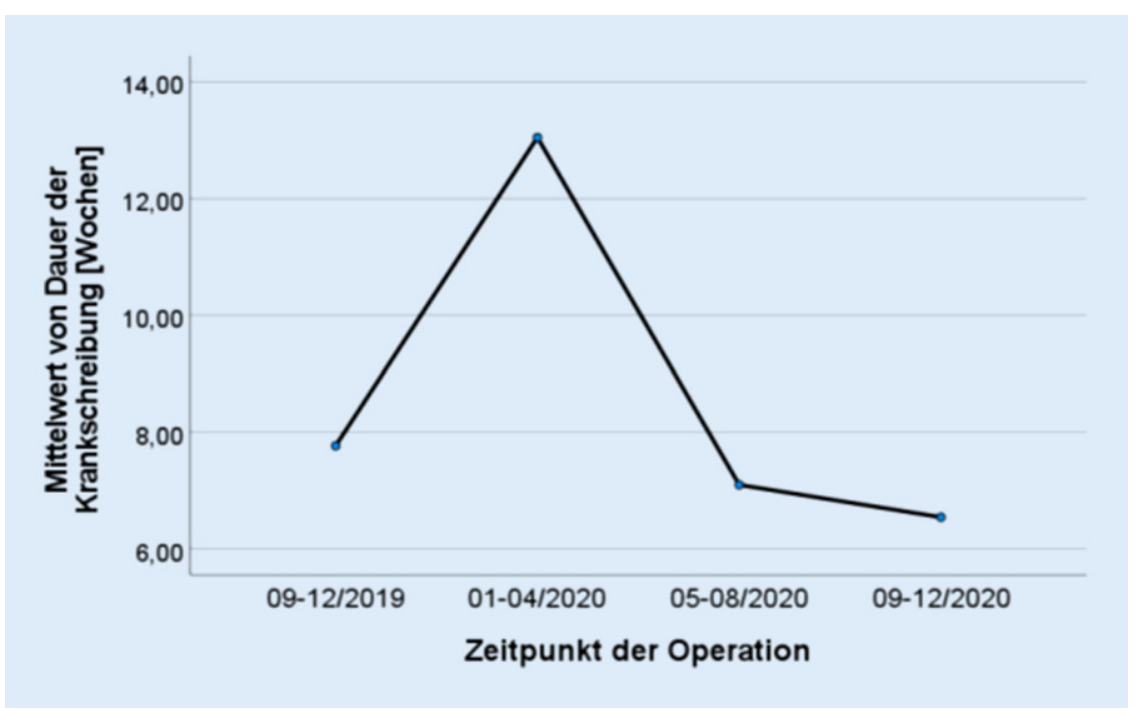

Abb. 1 A Durchschnittliche Dauer der postoperativen Arbeitsunfähigkeit bei Operation im letzten Tertial 2019 sowie im 1.-3. Tertial 2020

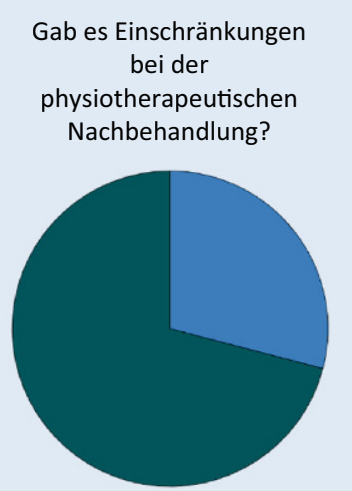

$$
\begin{aligned}
& \text { Hat Ihr Physiotherapeut } \\
& \text { Alternativen zum } \\
& \text { regulären Programm } \\
& \text { angeboten? }
\end{aligned}
$$

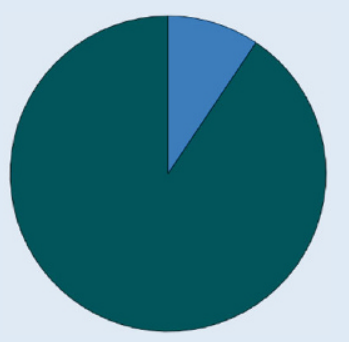

Hat Ihr niedergelassener Orthopäde Alternativen zum regulären Programm

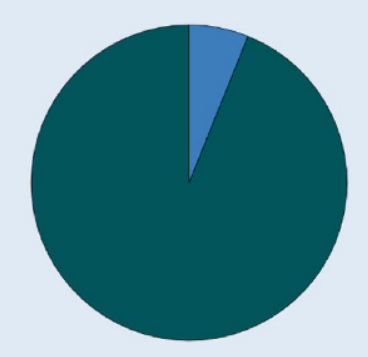

Gab es Einschränkungen bei der orthopädischen Nachbehandlung?

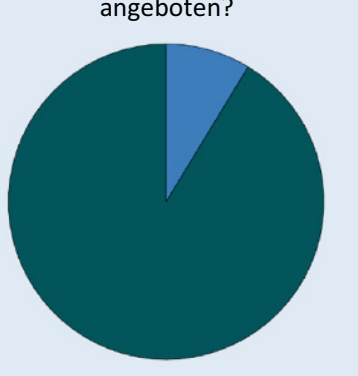
angeboten?

Würden Sie sich erneut in einer vergleichbaren Situation operieren lassen?

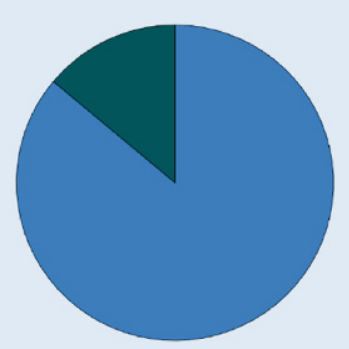

sicht der Arbeitsunfähigkeitsdauer in den einzelnen Tertialen ist in $\mathbf{A b b} 1$ dargestellt.

\section{Einschätzungen der Pandemie- auswirkungen durch die Patienten}

Von den 2020 operierten Patienten fühlten sich $39,8 \%$ in der physiotherapeutischen und $8,5 \%$ in der ärztlichen Nachbehandlung durch die COVID-19-Pandemie eingeschränkt ( $\bullet$ Abb. 2). Alternative Behandlungsoptionen wurden in der Physiotherapie 13,3\% der Patienten sowie in der Orthopädie 12,2\% der Patienten angeboten. $84 \%$ der Patienten würden sich erneut während einer vergleichbaren Situation operieren lassen.

\section{Diskussion}

Bei der vorliegenden Studie handelt es sich unseres Wissens um die erste Analyse der Auswirkungen der COVID-19-Pandemie auf die physiotherapeutische und ärztliche Nachsorge aus Patientensicht. Durchgeführt wurde die Befragung an Patienten nach einer Rekonstruktion des VKB. Diese Operation bietet in unseren Augen optimale Voraussetzungen für die Durchführung einer solchen Studie, da die Nachbehandlung stark standardisiert abläuft, was eine hohe Vergleichbarkeit sicherstellt. Zudem konnte für Rekonstruktionen des VKB bereits der hohe Stellenwert der physiotherapeutischen postoperativen Nachbehandlung für das langfristige Operationsergebnis belegt werden [7, 9-11]. Dennoch wollen wir an dieser Stelle betonen, dass sich ein großer Teil der Ergebnisse vermutlich auch auf andere Erkrankungen übertragen lässt, bei denen eine enge ärztliche und physiotherapeutische Nachbehandlung notwendig ist. Die Rekonstruktion des VKB wurde für die vorliegende Studie aus den o.g. Gründen stellvertretend als Beispiel gewählt. Insgesamt konnten wir zeigen, dass im Jahr 2020 im Zusammenhang mit der COVID-19-Pandemie die Wartezeiten auf Termine signifikant zunahmen, signifikant mehr Termine abgesagt wurden und es eine deutliche Tendenz zu einem verminderten Bewegungsumfang 3 Monate postoperativ gab. Gleichzeitig konnte dennoch durch eine Ausweitung der Möglichkeiten zur Arbeit im Homeof- 
fice die Dauer der postoperativen Arbeitsunfähigkeit bei vielen Patienten reduziert werden.

Hinsichtlich der physiotherapeutischen Nachsorge berichteten die Patienten von einer signifikant längeren Wartedauer auf Termine sowie auch einer höheren Anzahl abgesagter Termine und vergeblicher Terminanfragen. Folgerichtig fand auch der erste postoperative Termin zur Physiotherapie signifikant später statt $(1,15$ Wochen 2019 vs. 1,88 Wochen 2020). Dies bestätigt den allgemeinen Eindruck, dass die COVID-19-Pandemie einen relevanten Einfluss auf die postoperative Nachsorge hatte, obwohl Physiotherapie auch während aller "Lockdown"-Phasen als Teil der Grundversorgung weiter in Anspruch genommen werden konnten. Eine mögliche Erklärung für diese Auswirkungen bietet eine Onlineumfrage mit 1370 Therapeuten in der ambulanten Heilmittelversorgung, in der $88 \%$ der Teilnehmer eine höhere psychische Belastung aufgrund der COVID-19-Pandemie angaben, insbesondere aufgrund des zeitlichen Drucks durch die Hygienemaßnahmen, wirtschaftlichen Sorgen und Angst vor Infektion Angehöriger [12]. Interessanterweise steigerte sich die durchschnittliche Wartedauer auf Termine von 1,09 Wochen auf 2,57 Wochen um mehr als das doppelte, wohingegen es beim Zeitpunkt des ersten postoperativen Termins zu einer etwas geringeren Änderung von 1,15 auf 1,88 Wochen kam. Dies lässt vermuten, dass einige Patienten entsprechende Schwierigkeiten bereits vorausgesehen und sich daher früher als üblich um Termine gekümmert hatten.

Auch wenn bislang keine weitere Studie zu den Auswirkungen auf die physiotherapeutische Nachsorge nach VKBRekonstruktion in Deutschland vorliegt, bestätigen die international zu ähnlichen Fragestellungen veröffentlichten Studien diesen Eindruck, teils mit noch deutlicheren Unterschieden. So berichtet eine indische Studie, dass dortige Physiotherapeuten die Anzahl ihrer Patienten und die durchschnittliche Länge der Therapiesitzungen reduzieren mussten und sich durch das permanente Tragen der Schutzkleidung und die Infektionsangst physisch und psychisch beeinträchtigt fühlten [13]. In einer weiteren Studie aus Portugal bei 619 Physiotherapeuten gaben $73,2 \%$ an, dass sie pandemiebedingt keinen direkten Patientenkontakt mehr hatten und sich stattdessen oft für eine Therapie aus der Distanz mit schriftlichen Behandlungsverordnungen und synchroner Behandlung per Videokonferenz entschieden [13]. Im Vergleich dazu scheinen die Auswirkungen in Deutschland eher gering geblieben zu sein, was sich auch in der trotz der Einschränkungen hohen Zufriedenheit (2020 im Durchschnitt 7,8/10) niederschlägt.

Insgesamt sehr ähnliche Ergebnisse zeigten sich in unserer Studie hinsichtlich der ärztlichen Nachbehandlung. Auch hier kam es zu einer signifikanten Zunahme der Wartedauer auf Termine sowie der Anzahl vergeblicher Terminanfragen und abgesagter Termine mit einer entsprechenden Verzögerung des ersten postoperativen Nachsorgetermins. Im Gegensatz zur Physiotherapie gab es hier nur eine Tendenz zu etwas geringerer Zufriedenheit ohne signifikanten Unterschied zwischen 2019 und $2020(7,48 / 10$ vs. $7,16 / 10, p=0,4)$. Eine mögliche Erklärung hierfür ist der insgesamt deutlich intensivere Patientenkontakt während der physiotherapeutischen Behandlung, sodass Einschränkungen hier noch deutlicher wahrgenommen werden. Dazu passend gaben nur 8,5\% der Patienten an, sich bei der ärztlichen Nachbehandlung deutlich eingeschränkt gefühlt zu haben, wohingegen 39,8\% deutliche Einschränkungen in der physiotherapeutischen Nachsorge verspürten.

Neben den subjektiven Angaben der Patienten haben wir unseres Wissens als erste Studie auch die Auswirkungen auf das klinische Ergebnis in Form des postoperativen Bewegungsumfangs nach 3 Monaten untersucht. Hierbei zeigte sich bei den 2020 operierten Patienten eine deutliche Tendenz zu einem häufigeren Auftreten postoperativer Bewegungsdefizite gegenüber 2019, welche allerdings nicht signifikant war (Beugung: $10 \%$ vs. $23,3 \%, p=0,197$; Streckung: $4,3 \%$ vs. $18,8 \%, p=0,097)$. Auch wenn (mutmaßlich aufgrund des zu kleinen Patientenkollektivs) keine signifikanten Unterschiede gezeigt werden konnten, bestätigt die deutliche Tendenz unserer Meinung nach dennoch den hohen Stellenwert der postoperativen Nachsorge sowie die relevanten Auswirkungen der COVID-19-Pandemie.

Trotz der häufig geschilderten Einschränkungen in der Nachsorge gaben 34,9\% der 2020 operierten Patienten an, dass sich durch die im Rahmen der Pandemiebekämpfung erfolgte Ausweitung der Möglichkeiten zur Arbeit im Homeoffice die Dauer der Arbeitsunfähigkeit verkürzte. Im Vergleich mit den 2019 operierten Patienten gab es 2020 dennoch über das gesamte Jahr betrachtet eine Tendenz zu einer längeren Arbeitsunfähigkeit $(7,77$ Wochen vs. 8,53 Wochen, $p=0,688)$. Eine mögliche Erklärung bietet die genauere Betrachtung der Arbeitsunfähigkeitsdauer in den einzelnen Tertialen. Während es im ersten Tertial 2020 zu einem deutlichen Anstieg von 7,77 auf 13,04 Wochen kam, fiel die durchschnittliche Dauer bereits ab dem 2. Tertial mit 7,1 Wochen wieder deutlich ab und hielt sich auch im 3. Tertial mit 6,54 Wochen auf einem ähnlichen Niveau. Auch die einfaktorielle Varianzanalyse bestätigte signifikante Unterschiede in der Dauer der Arbeitsunfähigkeit zwischen den Tertialen $(p=0,048)$. Der Grund für diesen zeitlichen Verlauf ist wahrscheinlich darin begründet, dass es zu Beginn der Pandemie recht schnell zu deutlichen Einschränkungen in der Nachbehandlung mit entsprechend verlängerter Krankheitsdauer kam, wohingegen Arbeitgeber erst mit einer gewissen Latenz auf Angebote wie Homeoffice umstellten. Der hohe Anteil an Patienten, die eine Verkürzung der Arbeitsunfähigkeit angaben, zeigt eindrucksvoll das Potenzial solcher nun erstmalig großfächig eingeführten Maßnahmen.

In der physiotherapeutischen und ärztlichen Nachbehandlung hingegen wurden den Patienten nur relativ selten (Physiotherapie: 13,3\%; Orthopädie: 12,2\%) aufgrund der Pandemie Alternativen zum regulären Programm (z. B. Onlinetermine, Telefontermine) angeboten. Hier bieten sich daher mögliche Ansatzpunkte, um die Auswirkungen im Falle erneuter vergleichbarer Situationen weiter zu reduzieren. Dass hier noch dringender Nachholbedarf besteht, zeigt sich auch darin, dass sich immerhin 8,4\% der 2020 operierten Patienten aufgrund der pandemiebedingten Einschränkungen nicht erneut in einer 
vergleichbaren Situation operieren lassen würden.

Trotz der eindeutigen Ergebnisse unserer Studie sollten bei der Interpretation die Limitationen aufgrund des Studiendesigns beachtet werden. So handelt es sich um eine retrospektive Studie mit den üblichen Einschränkungen, wobei die beiden verglichenen Studiengruppen recht unterschiedlich große Kohorten beinhalten. Eine Vergrößerung der 2019er-Kohorte wäre allerdings nur durch den zusätzlichen Einschluss noch früher operierter Patienten möglich gewesen, was durch Abfrage weiter zurückliegender Erinnerungen unvermeidlich mit einer reduzierten Verlässlichkeit der Angaben verbunden gewesen wäre. Zudem unterliegen die Einschätzungen der Patienten zu großen Teilen keinen objektiven Kriterien, sondern vorwiegend subjektiven Wahrnehmungen. Die unterschiedlichen Kohortengrößen führen in erster Linie zu einer reduzierten statistischen Power, sodass eventuell nicht alle tatsächlich bestehenden Unterschiede zwischen den Gruppen tatsächlich nachgewiesen werden konnten, was z.B. auf unsere Beobachtungen zum Bewegungsdefizit zutreffen könnte.

\section{Fazit für die Praxis}

- In unserer Studie konnten wir zeigen, dass es durch die COVID-19(",coronavirus disease" 2019)-Pandemie und die dadurch bedingten Maßnahmen zur Kontaktbeschränkung zu signifikanten Einschränkungen sowohl in der physiotherapeutischen als auch in der ärztlichen Nachsorge gekommen ist, obwohl diese als Teil der Grundversorgung nie von einem „Lockdown" betroffen waren.

- Diese Einschränkungen schlugen sich auch in einer Tendenz zu häufigerem Auftreten von Beuge- und Streckdefiziten 3 Monate postoperativ nieder, auch wenn die Unterschiede nicht signifikant waren.

- Es besteht daher Bedarf an einem weiteren Ausbau alternativer Therapieoptionen, die bislang nur von ca. $13 \%$ der physiotherapeutischen und ärztlichen Praxen angeboten werden.

- Das große Potenzial solcher Angebote zeigt der große Erfolg der bisherigen Maßnahmen wie Homeoffice, durch die über ein Drittel der 2020 operierten Patienten die Dauer ihrer Arbeitsunfähigkeit subjektiv verkürzen konnten.

- Die vorliegende Untersuchung anhand von Rekonstruktionen des vorderen Kreuzbands sollte beispielhaft für eine Vielzahl

Influence of the COVID-19 pandemic on the physiotherapeutic and medical follow-up care after reconstructions of the anterior cruciate ligament

Background: The aim of this study was to examine the influence of the COVID19(coronavirus disease 2019) pandemic on the medical and physiotherapeutic followup care after reconstructions of the anterior cruciate ligament.

Methods: 116 patients ( 72 men and 44 women) who had received a reconstruction of the anterior cruciate ligament between September 2019 and December 2020 were included in this study. These patients were divided into two groups depending on the date of surgery: one group that had received surgery in 2019 before the COVID19 pandemic and one group that had undergone surgery in 2020 and was directly affected by the COVID-19 pandemic. All patients were interviewed using a standardized questionnaire regarding the influence of the coronavirus pandemic on the medical and physiotherapeutic follow-up care, as well as the moment of return to work. In addition, the range of motion of the respective knee 3 months postoperatively was analysed based on physical examination.

Results: At 3 months postoperatively, patients who had undergone surgery in 2020 showed a clear trend towards a higher frequency of extension deficits of $\geq 5^{\circ}(18.8 \%$ vs. $4.3 \%, p=0.097)$ or an inability to bend the knee $\geq 120^{\circ}(23.3 \%$ vs. $10 \%, p=0.197)$ compared to those who had received surgery in 2019. Patients who had undergone surgery in 2020 reported significantly longer delays for appointments, a higher number of futile attempts to get an appointment and a higher number of cancelled appointments, regarding both medical and physiotherapeutic follow-up care. $34.9 \%$ of the patients who received surgery in 2020 indicated that they were able to reduce the duration of their sick leave due to the increased possibilities of working in a home-office situation during the pandemic. Alternative treatment options due to the pandemic were offered by $13.3 \%$ of the physiotherapists and $12.2 \%$ of the physicians.

Conclusion: Although the physiotherapeutic and medical follow-up care was not directly affected by a "lockdown", the pandemic led to significant restrictions, which are also reflected in a clear trend towards worse clinical outcomes. Consequently, a further expansion of alternative treatment options, which were only offered by $12-13 \%$ of practices and that are presumably of comparable relevance for various other diseases, is needed.

\section{Keywords}

ACL reconstruktion · "Coronavirus disease" 2019 · Knee joint · Physical therapists · SARS-CoV-2

an Erkrankungen gesehen werden, für die ähnliche Auswirkungen zu vermuten sind.

\section{Korrespondenzadresse}

\section{Yannick Palmowski}

Center for Musculoskeletal Surgery, Charité Universitätsmedizin Berlin, HumboldtUniversität zu Berlin, Berlin Institute of Health Chariteplatz 1, 10117 Berlin, Deutschland yannick.palmowski@charite.de

Danksagung. Dr. Palmowski ist Teilnehmer am von der Charité - Universitätsmedizin Berlin und dem Berlin Institute of Health finanzierten BIH-Charité Junior Clinician Scientist Programm.
Funding. Open Access funding enabled and organized by Projekt DEAL.

\section{Einhaltung ethischer Richtlinien}

Interessenkonflikt. B. Bartek, T. Jung, A. Schwiedernoch, C. Perka und Y. Palmowski geben an, dass kein Interessenkonflikt besteht.

Alle beschriebenen Untersuchungen am Menschen oder an menschlichem Gewebe wurden mit Zustimmung der zuständigen Ethikkommission, im Einklang mit nationalem Recht sowie gemäß der Deklaration von Helsinki von 1975 (in der aktuellen, überarbeiteten Fassung) durchgeführt. Von allen beteiligten Patienten liegt eine Einverständniserklärung vor.

Open Access. Dieser Artikel wird unter der Creative Commons Namensnennung 4.0 International Lizenz veröffentlicht, welche die Nutzung, Vervielfältigung, Bearbeitung, Verbreitung und Wiedergabe in jeglichem Medium und Format erlaubt, sofern Sie den/die 
ursprünglichen Autor(en) und die Quelle ordnungsgemäß nennen, einen Link zur Creative Commons Lizenz beifügen und angeben, ob Änderungen vorgenommen wurden.

Die in diesem Artikel enthaltenen Bilder und sonstiges Drittmaterial unterliegen ebenfalls der genannten Creative Commons Lizenz, sofern sich aus der Abbildungslegende nichts anderes ergibt. Sofern das betreffende Material nicht unter der genannten Creative Commons Lizenz steht und die betreffende Handlung nicht nach gesetzlichen Vorschriften erlaubt ist, ist für die oben aufgeführten Weiterverwendungen des Materials die Einwilligung des jeweiligen Rechteinhabers einzuholen.

Weitere Details zur Lizenz entnehmen Sie bitte der Lizenzinformation auf http://creativecommons.org/ licenses/by/4.0/deed.de.

\section{Literatur}

1. Kohn L, Rembeck E, Rauch A (2020) Anterior cruciate ligament injury in adults : diagnostics and treatment. Orthopade 49(11):1013-1028

2. Mehl J et al (2018) Evidence-based concepts for prevention of knee and $A C L$ injuries. 2017 guidelines of the ligament committee of the German Knee Society (DKG). Arch Orthop Trauma Surg 138(1):51-61

3. Myklebust $\mathrm{G}$ et al (2003) Prevention of anterior cruciate ligament injuries in female team handball players: a prospective intervention study over three seasons. Clin JSport Med 13(2):71-78

4. AWMF (2018) S1 Leitlinie vordere Kreuzbandruptur. https://www.awmf.org/uploads/tx_ szleitlinien/012-005I_S1_Vordere_Kreuzbandrup tur 2019-02.pdf.Zugegriffen: 15. Febr. 2021

5. Herbort M GJ, Michel P, Domnick C, Fink C, Raschke MJ, Kittl C (2017) Aktuelle Techniken zur operativen Versorgung der Ruptur des vorderen Kreuzbandes. Sports Orthop Traumatol 33: p:367-378

6. Dragicevic-Cvjetkovic D et al (2014) The effects of rehabilitation protocol on functional recovery after anterior cruciate ligament reconstruction. Med Arch 68(5):350-352

7. van Grinsven S et al (2010) Evidence-based rehabilitation following anterior cruciate ligament reconstruction. Knee Surg Sports Traumatol Arthrosc 18(8):1128-1144

8. Gesundheit, B.f. (2021) Coronavirus-Pandemie (SARS-CoV-2): Chronik bisheriger Maßnahmen und Ereignisse. https://www. bundesgesundheitsministerium.de/coronavirus/ chronik-coronavirus.html?stand=20210217\%2F. Zugegriffen: 10. Aug. 2021

9. Kruse LM, Gray B, Wright RW (2012) Rehabilitation after anterior cruciate ligament reconstruction: a systematic review. J Bone Joint Surg Am 94(19):1737-1748

10. van Melick $\mathrm{N}$ et al (2016) Evidence-based clinical practice update: practice guidelines for anterior cruciate ligament rehabilitation based on a systematic review and multidisciplinary consensus. Br J Sports Med 50(24):1506-1515

11. Filbay SR, Grindem H (2019) Evidence-based recommendations for the management of anterior cruciate ligament $(\mathrm{ACL})$ rupture. Best Pract Res Clin Rheumatol 33(1):33-47

12. TAL gGmbH (2020) Aktuelle Auswirkung der Corona-Pandemie auf die ambulante Heilmittel- versorgung - Ergebnisse einer Online Befragung. https://tal-ggmbh.de/ergebnisse-der-onlinebefragung-zur-situation-in-der-ambulantenheilmittelversorgung-november-2020/.Zugegriffen: 10. Aug. 2021 (Zeitschrift für Physiotherapeuten)

13. MohitV, ShethM(2021)Changes in Clinical Practice in Physiotherapy as Repercussions of COVID-19 Pandemic. Int J Health Sci Res 11(3):2-8

\section{Cieditorial
Manager}

\section{Hilfestellungen für den Editorial Manager}

Das Einreichungs- und Begutachtungssystem Ihrer Zeitschrift

Sowohl für die ganz alltäglichen Fragen in der Handhabung des Editorial Managers als auch für spezielle Problematiken finden Sie auf www.springermedizin.de/editorialmanager eine Vielzahl an Handreichungen, die Ihnen die Arbeit als Gutachter*in, Autor*in oder Herausgeber*in erleichtern.

Über Videos, einseitige Schritt-für-SchrittAnleitungen oder ein umfangreiches Manual werden Sie durch die einzelnen Punkte geführt, wie:

- Wie reiche ich ein Manuskript ein?

- Wie finde ich passende Gutachter*innen?

- Wie lade ich Gutachter*innen ein?

- Wie nehme ich ein Gutachten an bzw. lehne es ab?

- Wo erkenne ich, in welchem Status ein Mansukript ist?

- Wie ändere ich meine persönlichen Informationen?

- Wo kann ich meinen Urlaub eintragen?

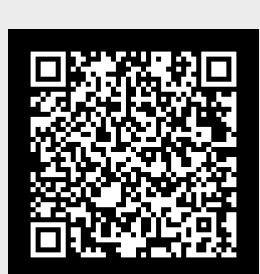

